\title{
Effect of Temperature on the Structure, Electrical Resistivity, and Charge Capacitance of Supported Lipid Bilayers
}

Shiju Abraham ${ }^{1}$, Tabea Heckenthaler ${ }^{1}$, Yakov Morgenstern ${ }^{1}$, Yair Kaufman ${ }^{1,2, *}$

${ }^{1}$ The Zuckerberg Institute for Water Research, The Jacob Blaustein Institutes for Desert

Research, Ben-Gurion University of the Negev, Sde Boqer 8499000, Israel.

${ }^{2}$ Currently at the Center of BioEngineering, University of California Santa Barbara,

California, United States of America.

*Corresponding author: Yair Kaufman, yairkauf@ucsb.edu.

\section{Supporting Information}

\section{Calculation of the effect of temperature on the lipid bilayer resistivity}

To calculate the changes (not absolute value) of the lipid bilayer resistivity as the temperature changes, $R_{\mathrm{LB}}(T)$, it is assumed that the rate limiting step (the slowest step) for ion transport through the lipid bilayer is the penetration of ions into the hydrophobic lipid bilayer core. According to Boltzmann distribution, the ion penetration rate, which is the lipid bilayer conductance, $P\left(\mathrm{~S}^{\cdot} \mathrm{cm}^{-2}\right)$, is proportional the probability for ions to penetrate a lipid bilayer:

$$
P(T) \propto \exp \left(-\frac{\Delta E(T)}{k T}\right)
$$

where $\Delta E(T)$ is the energy that is required to transfer an ion from the aqueous phase to the center of the hydrophobic core of the lipid bilayer, $k$ is the Boltzmann constant, and $T$ is the absolute temperature.

Assuming an uncharged lipid bilayer, $\Delta E$ is given by: ${ }^{1}$

$$
\Delta E(T)=\frac{e^{2}}{8 \pi \epsilon_{0} a}\left(\frac{1}{\epsilon_{\mathrm{LB}}}-\frac{1}{\epsilon_{\mathrm{W}}}\right)-\frac{e^{2}}{4 \pi \epsilon_{0} \epsilon_{\mathrm{LB}} h} \ln \left(\frac{2 \epsilon_{\mathrm{W}}}{\epsilon_{\mathrm{W}}+\epsilon_{\mathrm{LB}}}\right)
$$

where $e$ is the charge of electron, $\epsilon_{0}$ is the vacuum permittivity, $\epsilon_{\mathrm{LB}}$, and $\epsilon_{\mathrm{W}}$ are the dielectric constants of the lipid bilayer and the water, respectively, $a$ is the radius of an ion, and $h$ is the thickness of the lipid bilayer.

Lastly, the resistivity of the lipid bilayer is proportional to:

$$
R_{\mathrm{LB}}(T)=\frac{1}{P} \propto \exp \left(\frac{\Delta E(T)}{k T}\right)
$$

Therefore, the change (factor) in resistivity as the temperature changes is given by: 


$$
\frac{R_{\mathrm{LB}}(T)}{R_{\mathrm{LB}}\left(T_{0}\right)}=\frac{\exp \left(\frac{\Delta E(T)}{k T}\right)}{\exp \left(\frac{\Delta E\left(T_{0}\right)}{k T}\right)}
$$

\section{Effect of temperature on the topography of lipid bilayers without cholesterol}

As discussed in the main text, the effect of temperature on the topography of the lipid bilayers with or without cholesterol are similar. Fig. S2 shows fluorescence and AFM images that support this argument.

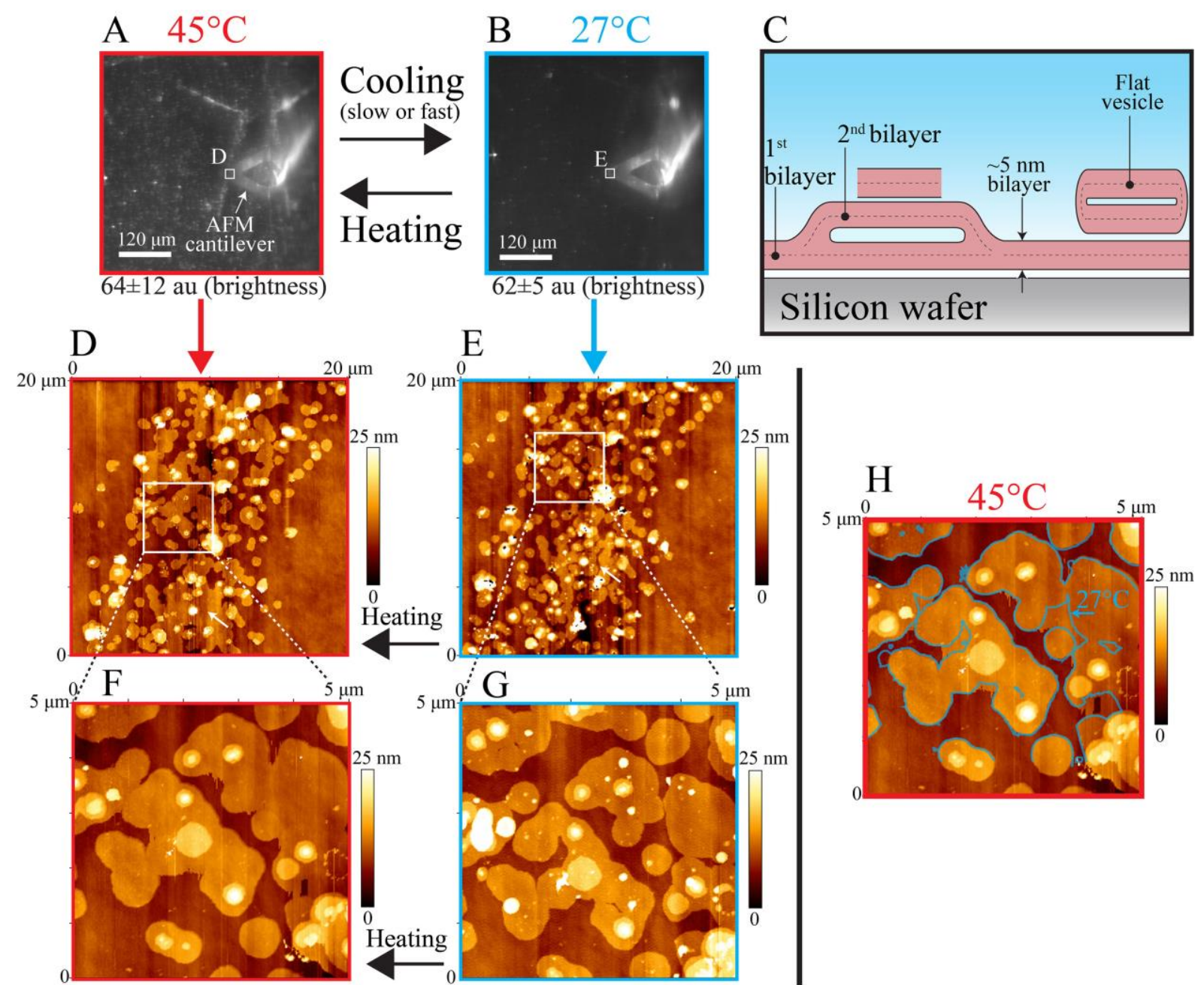

Figure S1: Effect of temperature changes on the topography of lipid bilayers without cholesterol. (A) Fluorescence image of the lipid bilayer at $45^{\circ} \mathrm{C}$, and (B) at $27^{\circ} \mathrm{C}$. The average fluorescence intensity, at the two temperatures, was similar, but bright objects $\left(2^{\text {nd }}\right.$ bilayer and intact vesicles, as depicted in (C)) were more visible at $45^{\circ} \mathrm{C}$. (D to G) AFM topography images show that the $2^{\text {nd }}$ lipid bilayer contracted and expanded upon cooling or heating. (H) Identical to $(\mathrm{F})$ and the bright blue line is the trace of $(\mathrm{G})$, emphasizing the expansion of $2^{\text {nd }}$ lipid bilayer upon heating. 


\section{References}

(1) Dilger, J. P.; McLaughlin, S. G.; McIntosh, T. J.; Simon, S. A. The Dielectric Constant of Phospholipid Bilayers and the Permeability of Membranes to Ions. Science 1979, 206 (4423), 1196-1198. 